\title{
THE STATUS OF ALPHA-PARTICLE DIAGNOSTICS
}

PPFL-CPP- -2622

\section{KENNETH M. YOUNG and DAVID W. JOHNSON}

Princeton Plasma Physics Laboratory, Princeton University, P.O.Box 451, Princeton, New Jersey, USA, 08543

\section{ABSTRACT}

There is a flurry of activity to complete alpha-particle diagnostics so that they can undergo some experimental testing in DT piasmas on JET or TFTR prior to implementation on ITER. Successful measurements of escaping charged fusion products have been made in DD plasmas, and the o-particle source can be well characterized by neutron profile measurement. These methods can be extrapolated to DT plasmas. Measurement of the confined a-particles requires a new technique. Collective 'Thomson scattering, methods involving charge-exchange interactions and nuclear reactions with impurities will be discussed. Some tssessment is given of the capabilities of these techniques, bearing in mind the potential for their use in the physics phase of the I'TER program.

\section{KEYWORDS}

$\alpha$-particles; fusion product particles; tokamaks; TFTR; collective Thomson scattering; neutral particle analysis; charge-exchange recombination spectroscopy,

\section{INTRODUCTION}

The only planned experiments in $\alpha$-particle physics prior to ITER will be completed in the next few years. The TFPR program will be completed in 1994 and that of JE'T in 1996 . These experiments will be able to validate many aspects of single particle behavior, some aspects of collective $\alpha$-particle effects but will not address heating by the $\alpha$-particles. Some experiments in simulating $\alpha$-particle behavior have already been done, in observing escaping charged fusion products and in generating the type of instabilities associatod with a very high energy group of jons. These two experiments also offer the only opportunity to demonstrate the physics of the methods proposed to measure the $\alpha$-particles directly. For ITER, the measurement will be critical, and because of its schedule and complex access, it is imperative that the physics aspects of the measurement techniques to be used have been fully demonstrated and that the instrumentation components are fully tested. A system of diagnostics is needed that can follow the temporal and spatial behavior of the $\alpha$-particles, from birth in the core of the plasma to the thermalized ash and loss to the divertor"s.

The $3.5 \mathrm{MeV} \alpha$-particles are the confined particles in the fusion reaction of deuterons and tritons. High energy fusion products are already observed in plasmas using deuterium fue! only. Here $2.5 \mathrm{MeV}$ neutrons, $0.8 \mathrm{MeV} 3 \mathrm{He}$ ions, $3.0 \mathrm{MeV}$ protons and $1.0 \mathrm{MeV}$ tritons are created. Measurements of the neutrons and escaping charged fusion products are alxeady playing an important role in understanding the plasma behavior. "The "burnup" of the confined tritons, and herice the radial diffusion of these ions, can be followed by measuring the $14 \mathrm{MeV}$ neutrons produced in their fusion reactions with the background deuterons. Deuterons and $3 \mathrm{He}$ ions, sometimes used in minority heating with ion cyclotron resonance heating, also fuse to give a $3.7 \mathrm{MeV}$ (y.particle and a $15 \mathrm{MeV}$ proton.

Diagnostics of $\alpha$-particles have been discussed for some years. The methods belong in two main families, some depending on collective Thomson scattering, others tepending on $\alpha$-particle collisions with neutral particles to create escaping He atoms, radiation of $H_{e}$ spectral lines or nuclear product.s. The former methods do not perturb the plasma and can 


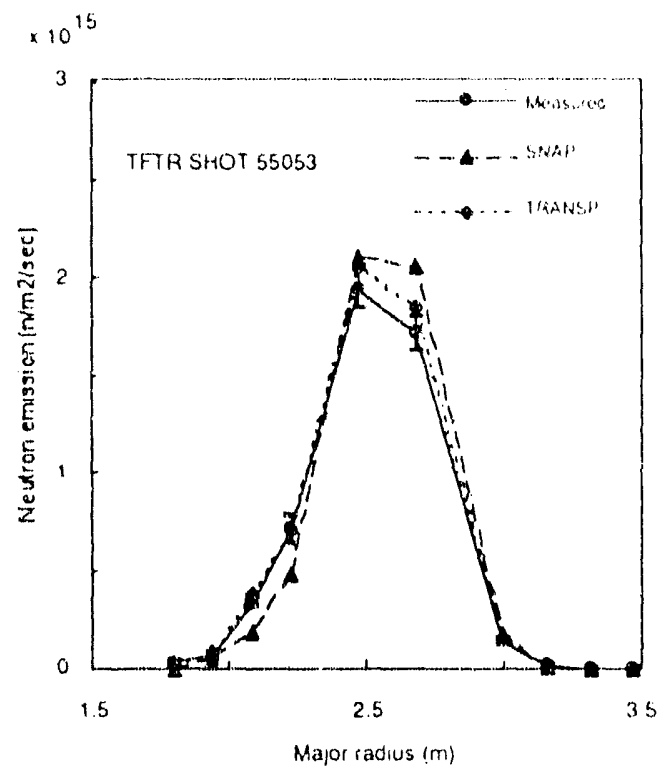

Fig. 1. The shape of the measured neutron flux of a 'TF'IR supershot is compared to the neutron fluxes predicted from the SNAP and TRANSP transport codes using other plasma measurements.

have access to the plasma center, also the peak of the $\alpha$-particle source. However, high power pulsed sources are necessary because of the low cross-section, their spatial resolution is not good and the plasma background signal may be high, particularly at higher frequency. The neutral-particle methods require either a high energy neutral impurity beam or an injected impurity pellet to provide the target particles. They provide relatively good spatial resolution and the possibility of determining the full energy spectrum. Both of these methods have difficulty penetrating to the center of large, hot plasmas and perturb the plasma to some extent.

Measurement of the escaping charged fusion products has been made on a number of tokamaks revealing important physics information about the confinement of the highenergy particles. Recent reports from TFTR have included measurement of diffusion, the effects of toroidal field ripple, and the impact of MHD activity in the plasma core.

This paper will describe these methods briefly. For further details the reader is referred to recent Review articles (Young, 1991, Zweben, 1986) and references given there.

\section{ALPHA SOURCE MEASUREMENT}

The $\alpha$-particle source will be obtained with relatively high accuracy using a multichannel array of neutron detectors. These detectors view through long tubes in thick shielding material and are themselves very highly shielded so that their neutron signals arise only from a collimated region in the plasma and are not affected by scaltered neutrons. Such measurements are done routinely on TFTR (Johnson et al., 1992, Roquemore et al., 1990) and JET (Jarvis et al.,1990) for DD plasmas and were a key measurement in the JET preliminary tritium experiment (JET Team, 1992). The only limitation on the quality of this measurement is the availability of access for a spread-array of collimator pipes.

Figure 1 shows an experimental profile for a beam heated supershot plasma in TFTR (Johnson et al., 1992). There are ten active viewing channels looking up from below, each with a $\mathrm{ZnS}(\mathrm{Ag})$ plastic scintillator for DD neutron detection. The detectors have been independently calibrated using a ${ }^{252} \mathrm{Cf}$ neutron source in the vacuum vessel. The measured data points are compared with the results of transport code calculations making use of the spatial dependence of other plasma parameters. These codes are the equilibrium transport code SNAP and the time dependent code TRANSP. This good agreement is complemented by a similar agreement over the length of a plasma pulse between the integral of the neutrons measured by the collimator and a calibrated system of neutron flux monitors. 
ESCAPING ALPHA-PARTICLES

Lost-a Probes

Escaping fusion product particles have been measured on many tokamaks, among them PLT, ASDEX, TFTR, JET, DIII.D and T-10 (Zweben et al., 1990A and references therein). Most of these measurements have been made with solid-state detectors, which cannot survive in high neutron fluences. The thin scintillators used on TFTR (Zweben) or Faraday cups proposed for JET (Conroy et al., 1991) are planned for use for o-particles from the D'T experiments. The scintillators are mounted behind an aperture outside the last closed flux surface, at the bottom of the vacuum vessel, for observing first-orbit losses, or near the outer midplane for looking at loss in the ripple of the toroidal field.

In studies of the protons and tritons from DI) plasmas in THTR, it has already been possible to identify some important features: first-orbit losses; toroidal field ripple-induced diffusion of trapped $\alpha$-particle orbits; ripple-trapped $\alpha$-particles in the magnetic wells between toroidal field coils; losses due to MHD instabilities; and beam-ion loss when TAE modes were present. Extrapolating these probes to an ignited tokamak is important but difficult (Zweben et al., 1990B). The probes necessarily have to be close to the plasma and therefore sustain very high heat loads, high neutron fluxes and possibly intense integrated $\alpha$-particle energy. In TFTR, the probes have to be protected by thick carbon-carbon composite shields and are actively cooled. For ITER, where the predominant loss mechanism is potentially due to ripple trapping, these probes would have to be integrated into the first wall. Development of scintillators able to operate at higher temperatures and survive in the high radiation environment will be required. Alternative detectors, such as Faraday cups, local infra-red first-wall temperature measurement, readily-removable surface samples or nuclear reactions in beryllium targets could be considered.

\section{Ion Cyclotron Emission}

A very interesting observation during the JET preliminary tritium experiment (JET Team, 1992) was that the intensity of the second harmonic of the ion-cyclotron emission increased linearly with the neutron rate over six orders of magnitude (Cottrell, 1992). Spectra in the ion cyclotron frequency range have been seen for many years in DD plasmas in both JET (Cottrell and Dendy, 1988) and 'FFT (Greene et $a l ., 1990$ ) and have been related to highenergy beam ions or to charged fusion particles. 'There are some differences in the observed spectra, but the most intriguing common feature is that the harmonic separation is that for the toroidal field strength at the outside (low-field) scrapenff region. The JET measurement is made with a large ion-heating antenna at the horizontal mid-piane and the TFTR measurement is done with small loops mounted above and below the plasma, near the vertical rnid-plane. These results are very suggestive of a process associated with escaping high-energy particles, but no convincing mechanism has yet been put forward. 'This is a non-intrusive measurement, the detectors can be simple and able to be quite distant from the plasma, and so it is important to find an explanation for the emission that can be used to better characterize the $\alpha$-particle behavior.

\section{CONFINED ALPHA-PARTICLES}

\section{Cellective Thomson Scattering}

Using an intense coherent source, collective Thomson scattering from shielding electrons moving with the $\alpha$-particles can be observed (Hughes and Smith et al., 1987). Scattering off thermal ions has been experimentally demonstrated (Nieswand et al., 1991). Complex scattering systems are now being built for the DT phases of JET (Costley et al., 1988) and TFTR (Woskov et al., 1988) for measurement of the $\alpha$-particle energy distribution with limited spatial capability. Both of these systems will operate in the microwave range. Alternatively a $10 \mu \mathrm{CO}_{2}$ laser could be used (Kruglyakov et al., 1991, Richards et al., 1992) and, for ITER, a frequency of 1.5 THz has been proposed (Mukhovatov et al., 1991).

An ideal calculated spectrum for the scattering in I'TER for the 1.5 THz system is shown in Fig. 2 for one of the reference ITER plasmas. The figure shows the contributions to the overall scattered signal from the electrons, thermal ions and $\alpha$-particles. The width of the ion feature sets the lower energy limit that can be measured for the $\alpha$-particles.

JET will use O-mode incoming waves, with frequency between the fundamental and first harmonic of the ion cyclotron frequency. TFTR will use X-mode incoming waves with 


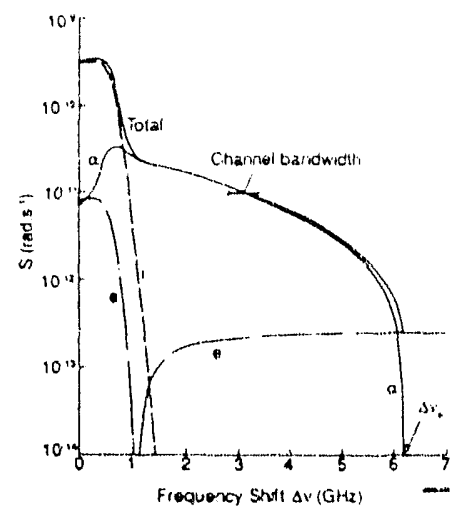

Fig 2. The scattered spectrum calculated for l'TER for a $1.5 \mathrm{TH}$ source. The contributions for electrons, thermal ions and $\alpha$-particles are shown. $\theta=5.5^{\circ}, n_{e}(0)=5 \times 10^{19} \mathrm{~m}^{-3}$, $\mathrm{n}_{\alpha}(0)=2 \times 10^{18} \mathrm{~m}^{-3}$ and $\mathrm{T}=10 \mathrm{keV}$.

frequency below the fundamental. There was some concern that the plasma backgrotind noise level may be significant at these frequencies, but in 'TFTR it was found to be equivalent to a black-body temperature of less than $20 \mathrm{eV}$ at the relevant $60 \mathrm{GHz}$ frequency.

These scattering systems are costly, and, because of the low scattering cross-section, require very high power pulsed sources. TFTR and JE'T will use gy rotron sources, the JET source now being in development. The 1.5 'THz source for ITER is clearly a major development issue, but it does allow for reasonably large scattering angles, with the concomitant spatial resolution and design options. However, the technology of $10 \mu$ systems is well advanced and the feasibility of doing collective scattering (the electron feature) in a tokamak has been demonstrated at $\mathrm{A}^{\prime} \mathrm{TF}$ (Richards et al, 1992). 'The scattering angle is small, s1. typically, making the spatial resolution poor and constraining the design options. It should be noted that refraction of the beam, and hence dependence on the plasma profile, becomes larger as the wavelength is increased; hence microwave suurces are not considerec feasible for the large, high density plasmas of ITER. Other alternative ideas for collective Thomson scattering making use of the enhancement of the spectral function due to plasma waves cannot be addressed.

\section{Neutral Particle Analysis}

Post (1981) proposed that neutralization of the high energy $\alpha$-particles by their exchanging charge with a neutral atom population could allow the $\alpha$-particles to be measured. The cross-section for this interaction is only significant if the particles have velocities of the same order of magnitude. A beam of neutral lithium atoms with energy $7 \mathrm{MeV}$ was proposed. This beam would both supply fast target atoms but also penetrate to the center of the fiasma. Such a system has not yet been built, because of the high cost of the beam development, but two alternate schemes are being actively pursued.

The first scheme makes use of a helium beam, one of the $120 \mathrm{kV}$ heating beams, on JET (Petrov et al., 1992). With $5 \mathrm{MW}$ of heating beam power, the number of neutral particles compensates sufficiently so that a good signal to noise ratio is predicted for a witie energy range of the slowing down $\alpha$-particles. The neutral particle analyzer is of the billB type. with eight energy channels. It can resolve $\mathrm{H}, \mathrm{D}, \mathrm{T}, \mathrm{T}$. He and $4 \mathrm{lle}$ atomic fluxes, a $400 \mathrm{~A}$ entrance foil stripping of an electron prior to the analysis. Its energy range is $0.5 \mathrm{MeV} \leq \mathrm{E} \leq 3.5 \mathrm{MeV}$. A prototype instrument has already been used in the measurement. of high energy hydrogen fluxes during ICRF minority hydrogen heating. T'he analyzer detector is made of thin CsI + 'Tl scintillators coupled to photomultipliers so that the sensitivity to high energy ions is almost $100 \%$ while the sensitivity to neutrons and gammas is very low. This last feature is essential for a measurement system for $\alpha$-particles.

An almost identical analyzer will be used on TFTR (Fisher et al., 1990); the source of neutral particles will be the ablation cloud of a lithium pellet fired into the plasma at a velocity of about $800 \mathrm{~m} / \mathrm{s}$. (Full penetration of the pellet to the plasma center would require a velocity of $-5 \mathrm{~km} / \mathrm{sec}$.$) . Where the \alpha$-particles exchange their charge, the density of $\mathrm{Li}^{+}$ions could be of order $10^{20} \mathrm{~cm}^{-3}$, this high density compensating for the low cross-section. Thus an 


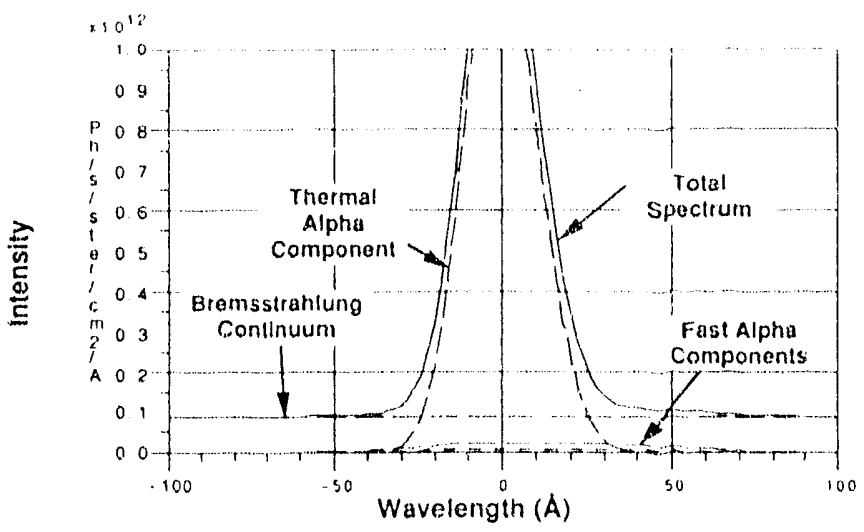

Fig. 3. Calculated helium $4686 \AA$ spectrum for an o-particle density of $1 \times 10^{12}$ ' $\mathrm{m}^{3}$ in a 'TF"TR supershot. $A$ TF'TR heating beam is the source of neutral particles.

analyzer aligned to look nearly parallel to the peilet flight path in the plasma, but several centimeters along the field from the pellet line, so that the analyzer observes the optimum region for $\mathrm{Li}^{+}$in the stretched-out ablation cloud, will receive a very high flux of neutralized $\alpha$-particles. The whole energy spectrum, down to the limit set by the entrance-foil thickness, is potentially available from such a measurement, but at only one instant in time, and, potentialiy, with considerable modification of the plasma. Carbon and boron pellets are under consideration to achieve better penetration with a sacrifice in count rate.

\section{Sepectroscopy of Helium Lines}

The atomic collision of an $\alpha$-particle with an incoming neutral atom may result in a singlycharged Het ion in an excited state, which will radiatively decay emitting lines in the helium spectrum (Post et al, 1981). To optimize the cross-section the atoms in an incoming neutral beam should have a similar velocity to the high-energy $\alpha$-particles. Alternatively, the signal strength can be enhanced using the high density of a pellet ablation cloud (Sasao et al., 1986). But the principle can be evaluated, at least for a-particle energies less than about $800 \mathrm{keV}$, using the heating beam particles in TF"TR or JE'T. 'These are deuterium neutral beams with energies aboui $100 \mathrm{keV}$.

Low energy helium transport has been extensively studied by injecting helium gas into deuterium plasmas and observing the charge-exchange helium spectra (Fonck et al., 1984, Synakowski et al., 1990, von Hellerman et al., 1990). But the density of helium is considerably higher in these experiments, and the energy range is much less than for the $\alpha$ particles in a D'T plasma where the $\alpha$-particle density may be $1 \%$ of the electron density. Figure 3 shows a calculated spectrum for a discharge in TF'IR. The visible $4686 \mathrm{~A}$ line was selected because of the ease of instrumentation and ability to use fiberoptic signal transmission. The spectrum shows an example of a calculated total helium spectrum for a relatively high $\alpha$-particle density of $1 \times 1012 \mathrm{~cm}^{3}$ superposed on the expected bremsstrahlung continuum in a TFTR supershot. In the calculation a beam density of $1 \times 10^{9} \mathrm{~cm}^{-3}$ was assumed and the actual energy species in the beam were included. A slowing-down $\alpha$-particle energy distribution was used. "The spectrum is dominated by the central thermal peak, but the tails on either side contain the information about the fast particles. The upper limit is set by the decay of the crossisection because of the velocity difference between the beam particles and the $\alpha$-particles. A very high throughput optical system couples five spatial channels to an $\mathrm{f} / 3.8$ spectrometer, each channel equipped with a low-noise CCD detector, in the implementation for the 'TFTR DT program (Stratton et al., 1992).

\section{Gamma_Spectroscopx}

Cecil et al. (1986) and Kiptilij (1990) independently proposed the use of gamma spectroscopy for $\gamma \mathrm{s}$ from nuclear reactions of the $\alpha$-particles with impurity nuclei as a measurement technique. In the former case, resonant reactions such as $7 \mathrm{Li}(\alpha, \gamma){ }^{11} \mathrm{BB}$ were proposed where a $\gamma$-spectrum will be produced according to the resonant states of the finalnuclei. The 
intensities will be proportional to the concentration of $\alpha$-particles that have slowed to the resonant energy. It appears likely, however, that the ratio of the peaks to the background signal arising from scattering of high energy neutrons may be too small for effective measurement. Kiptilij proposed using the ${ }^{9} \mathrm{Be}(\alpha, n \gamma){ }^{12} \mathrm{C}$ reaction which has a threshold $\alpha$. particle energy of $1.7 \mathrm{MeV}$. "The product $\gamma$-energy is $4.4 \mathrm{MeV}$. An analysis of the anticipated spectral broadening for various $\alpha$-particle energy distributions is required. The most. difficult aspect of this technique is the provision of sufficient shielding for the detector le.g. Ge(Li)] with a smal! collimator tube fifled with a neutron absurber, so that the very high neutron, and resultant scattered- $\gamma$ fluxes do not dominate the signal.

\section{ACKNOWLEDGEMENTS}

We are particularly grateful to our colleagues who have discussed their work with us and who have comnented on this Review paper. Note that the reference list is by no means comprehensive. This work was carried out under U.S. Department of Energy Contract No. DE-AC02-76-CHO3073.

\section{REFERENCES}

Cecil, F.E., S.JZweben and S.S. Medley (1986). A method for determining fast appha particie confinement in tokamak plasmas using resonant nuclear reactions. Nucl. Instrum. Methods Phy's. Rev., 245, 547-552.

Conroy, S.W., et al. (1991). Alpha-particle diagnostics for the D.T phase. Pruc. 18 th European Conf, on Contr. Fus. and Plasma Phys, LY, $265-268$.

Costley, A., et al. (1988). A Thomson scattering diagnostic to moasure fast ion and a-particle distributions in JET. JE' Report, JET-R(89)08.

Cottrell, G.A. and R.O.Dendy (1988). Superthermal radiation from fusion products in Jer. Phys. Rev. Lett., $60,33-36$.

Cottrell, G.A. (1992). Identification of ion cyclotron emission from fusion a-particles. Intern. Conf. on Plasma Phys., Innsbruck.

Fisher, R.K., et al. (1990). Fast alpha diagnostics using cambon pellet injection. Rev. Sci. Instrum., 61, 3196-

Fonck, R.J. and R.A. Hulse (1984). He transport in tte HDX tokanak. Phys. Rell. Lett. 52.2530-533.

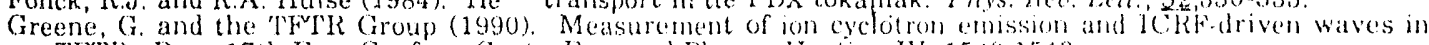

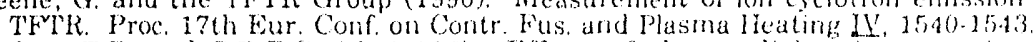

Hughes, T.P. and S.R.P.Smith (1987). Effects of plasma dielectric properties on 'I'homson scattering of millimetre waves in tokamak plasmas. Jour. Plasma Phys., 12 2 $215-210$

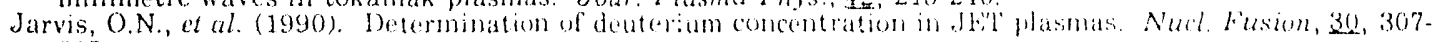

315 .
JET'Team (1992). Fusion energy production from a deuterium-tritium plasma in the Jef' tokamak. Nuclear Fusion, 32 187-203.

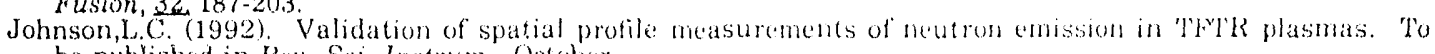
be published in Rev. Sci. Instrum., October.

Kiptilij, V.G. (1950). Capabilities of gamma spectroscopy for fast alpha-particle diagnostics. fius. Technology, 18, $583-590$.

Kruglyakov, E.P. (1991). Unconventional laser methods in plasma diagnostics. Diagnostics for Contemporary Fusion Experiments, Sucieta Italiana di Fisica, Bologna, Italy, 95-112

Mukhovatov, V., et al. (1991). I'TER Diagnostics, ITER Documentation Series No. 33 (IAEA, Vienna); ITER

operation and diagnostics. Nucl. Fusion Supplement $3,427-442$.
Nieswand, C., et al., (1991). Measurement of ion temperature profiles in the Ten tokamak by collective ieswand, C., et al., (1991). Measurement of ion temperature profiles in the TCA tokamak by col
Thomson scattering. Proc. 18th European Conf. on Contr. Fusion and Plasma Phys., IV, 289-292.

Petrov, M.P., et al. (1992). Neutral Particle Analysis in the MeV Range in JET. Interi. Conf. on Plasma Phys, Innsbruck.

Post, D., et al. (1981). Techniques for measuring the alpha-particle distribution in magnetically confined plasmas. Journal of Fusion Energy, 1, 129-42.

Richards, R.K. et al. (1992). Measurement of the CO2 laser small-angle scattering on a magnetically confined plasma. Submitted to Phys. Rev. Letl.

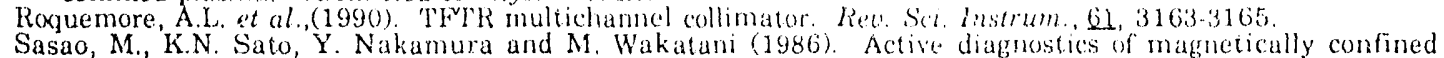
alpha particles by pellet injection. Proceedings of the Course "Basic and Advanced Diagnostic Techniques for Fusion Plasmas," Varema, Italy, Vol Lh, 775-780.

Stratton, B.C., et al. (1992). Alpha-CHERS: a spectroscopic experiment to detrect non-thermal aipha particles on TFTR. To be published in Reu. Sci. lnstrum. October.

Synakowski, E.J. et al. (1990). Measurement of radial profiles of He2t transport coefficionts on the TFTR tokamak. Phys. Rev. Lett. $05,2255-2258$

von Hellerman, M., et al. (1990). Visible charge exchange spectroscopy at Jer. Re'. Sci. lnstrum.,61, 34793486.

Woskov, P., et al. (1988) Gyrotron collective Thomson scattering diagnostics for confined alpha particles in TFTR. Rev. Sci. Instrum., 59, 1565-1567.

Young, K.M. (1991). Alpha particle diagnostics. Diagnostics for Contemporary Fusion Experiments, Societa Italiana di Fisica, Bologna, Italy, 573.594

Zweben, S.J. (1986). Approaches to the Diagnostics of alpha particles in tokamaks. Rev. Sci. Instrum. 57. $1723-1728$.

Zweben, S.J., et al. (1990A). Loss of alpha-like MeV fusion products from 'JFTR. Nucl. Fusion, 30, $1551-1574$.

Zweben, S.J., et al. (1990B). Constraints on escaping alpha particle detectors for ignited tokamaks. Reu. Sci. Instrum., 61, $3505-3508$ 


\section{DISCLAIMER}

This report was prepared as an account of work sponsored by an agency of the United States Government. Neither the United States Government nor any agency thereof, nor any of their employees, makes any warranty, express or implied, or assumes any legal liability or responsibility for the accuracy, completeness, or usefulness of any information, apparatus, product, or process disclosed, or represents that its use would not infringe privately owned rights. Reference berein to any specific commercial producl, process, or service by trade name, trademark, manufacturer, or otherwise does not necessarily constitute or imply its endorsement, recommendation, or favoring by the United States Government or any agency thereof. The views and opinions of authors expressed herein do not necessarily state or reflect those of the United States Government or any agency thereof. 

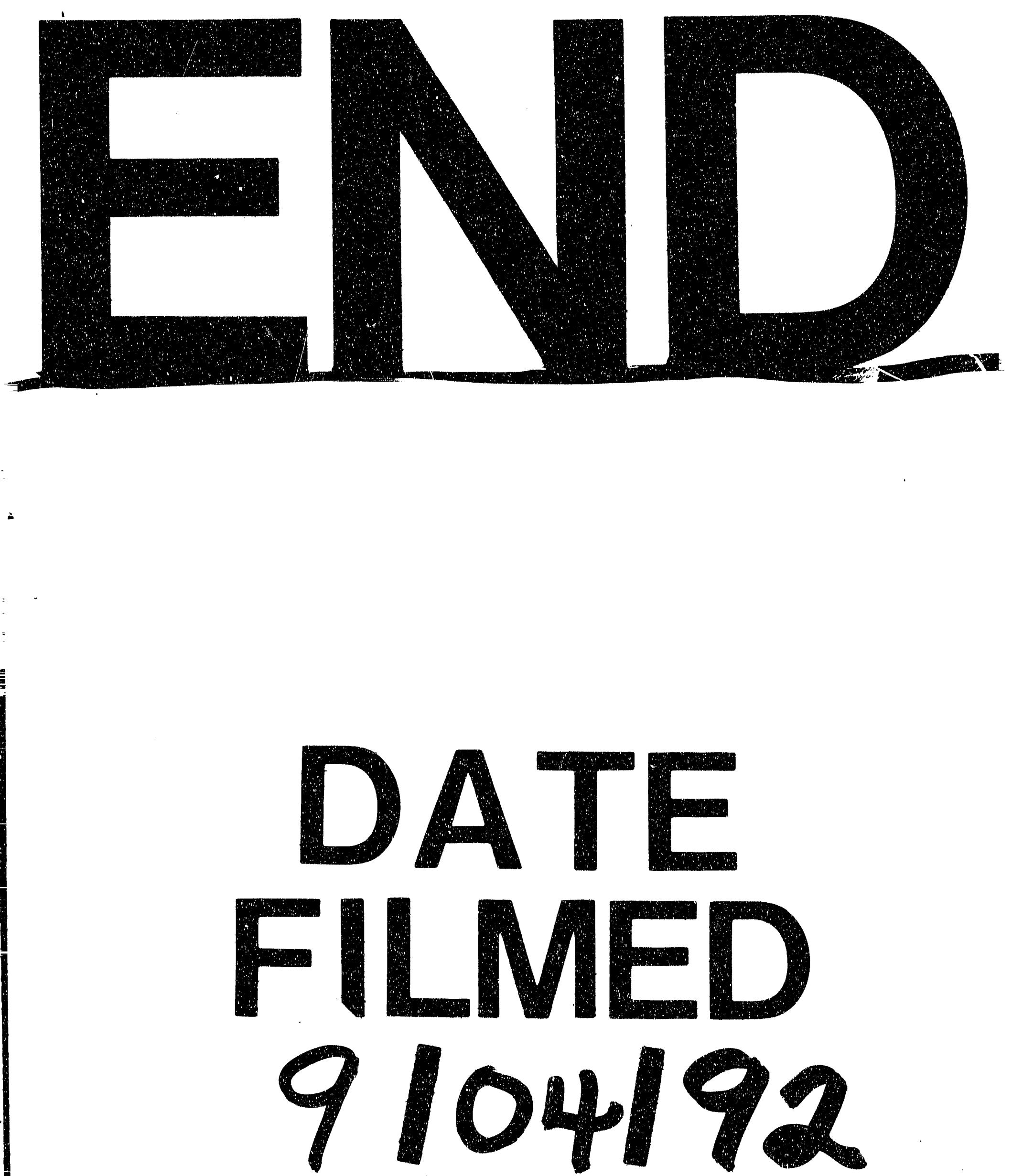
